\title{
Comparing parasitological $v s$ serological determination of Schistosoma haematobium infection prevalence in preschool and primary school-aged children: implications for control programmes
}

\author{
WELCOME M. WAMI ${ }^{1,4} *$, NORMAN NAUSCH ${ }^{1,4}$, KATHARINA BAUER ${ }^{1,4} \dagger$, NICHOLAS \\ MIDZI $^{2} \ddagger$, REGGIS GWISAI ${ }^{3}$, PETER SIMMONDS ${ }^{4}$, TAKAFIRA MDULUZA ${ }^{5}$, MARK \\ WOOLHOUSE $^{4}$ and FRANCISCA MUTAPI ${ }^{1,4}$ \\ ${ }^{1}$ Institute of Immunology $\Xi^{\circ}$ Infection Research, University of Edinburgh, Ashworth Laboratories, King's Buildings, \\ West Mains Rd, Edinburgh EH9 3FT, UK \\ ${ }^{2}$ National Institute of Health Research, P.O. Box CY 573, Causeway, Harare, Zimbabwe \\ ${ }^{3}$ Ministry of Health and Child Care, Murewa District Hospital, P.O. Box 60, Murewa, Zimbabwe \\ ${ }^{4}$ Centre for Immunity, Infection Eळ Evolution, University of Edinburgh, Ashworth Laboratories, King's Buildings, \\ West Mains Rd, Edinburgh EH9 3FT, UK \\ ${ }^{5}$ University of Zimbabwe, Biochemistry Department, P.O. Box MP167, Mount Pleasant, Harare, Zimbabwe
}

(Received 18 December 2013; revised 24 Fanuary 2014; accepted 29 Fanuary 2014; first published online 28 March 2014)

SUMMARY

To combat schistosomiasis, the World Health Organization (WHO) recommends that infection levels are determined prior to designing and implementing control programmes, as the treatment regimens depend on the population infection prevalence. However, the sensitivity of the parasitological infection diagnostic method is less reliable when infection levels are low. The aim of this study was to compare levels of Schistosoma haematobium infection obtained by the parasitological method vs serological technique. Infection levels in preschool and primary school-aged children and their implications for control programmes were also investigated. Infection prevalence based on serology was significantly higher compared with that based on parasitology for both age groups. The difference between infection levels obtained using the two methods increased with age. Consequentially, in line with the WHO guidelines, the serological method suggested a more frequent treatment regimen for this population compared with that implied by the parasitological method. These findings highlighted the presence of infection in children aged $\leqslant 5$ years, further reiterating the need for their inclusion in control programmes. Furthermore, this study demonstrated the importance of using sensitive diagnostic methods as this has implications on the required intervention controls for the population.

Key words: parasitology, serology, prevalence, schistosomiasis, diagnosis, neglected tropical diseases.

\section{INTRODUCTION}

Urogenital schistosomiasis is a waterborne disease caused by infection with Schistosoma haematobium and is a major public health problem among poor communities in sub-Saharan Africa (Gryseels et al. 2006; Kabatereine et al. 2007; WHO, 2012). Eggs laid by adult female $S$. haematobium worms are excreted through urine, inflicting damage to the genitourinary tract. Children living in endemic areas

\footnotetext{
* Corresponding author: Institute of Immunology \& Infection Research, School of Biological Sciences, University of Edinburgh, Ashworth, Laboratories, King's Buildings, West Mains Rd, Edinburgh EH9 3JT, UK.E-mail: W.M.Wami@sms.ed.ac.uk

† Current address: German Cancer Research Center (DKFZ), Im Neuenheimer Feld 280, 69120 Heidelberg, Germany.

\$ Current address: University of Zimbabwe, College of Health Sciences, Department of Medical, Microbiology, P.O. Box A178, Avondale, Harare, Zimbabwe.
}

tend to carry the highest disease burden (Hotez et al. 2006; Stothard et al. 2011a) and symptoms of urogenital schistosomiasis amongst these children are commonly characterized by the presence of blood in urine (haematuria) and painful urination (van der Werf et al. 2003; Sady et al. 2013). Chronic infection results in severe pathologies such as kidney failure and urinary tract and bladder wall fibrosis. Other symptoms include malnutrition, stunted growth and impaired memory and cognition (Pasvol and Hoffman, 2001; Sousa-Figueiredo et al. 2008; WHO, 2010; Muller et al. 2011).

The infection and its associated morbidity can be controlled with chemotherapy using praziquantel (Doenhoff et al. 2008; Mutapi et al. 2011), administered at a standard oral dosage of $40 \mathrm{mg} \mathrm{kg}^{-1}$ body weight (WHO, 2002). Praziquantel is safe and efficacious in children aged 5 years or under (Mutapi et al. 2011; Stothard et al. 2011b; Coulibaly et al. 2012), but so far treatment of children belonging to 
this age group has not yet been fully integrated into the control programmes (Ekpo et al. 2012). Preschool-aged ( $\leqslant 5$ years) children have been neglected both in terms of research and in control programmes for the previously held view that they carry insignificant infection levels (Stothard and Gabrielli, 2007; Mutapi et al. 2011; WHO, 2011). This was further exacerbated by poor diagnosis of infection in the field (Vennervald et al. 2000; Stothard et al. 2011a). The exclusion of preschoolaged children from current control programmes increases their risk of developing future morbidity (Stothard and Gabrielli, 2007; Sousa-Figueiredo et al. 2008) and also indicates that disease burden in this age group is still not well defined (Garba et al. 2010). Consequently, this may have negative impacts on the overall effectiveness of control programmes.

In line with the guidelines outlined by the World Health Organization (WHO, 2002), infection prevalence must be determined prior to the implementation of a control programme (Dawson et al. 2013; WHO, 2013). To ensure that infection transmission levels are reduced and the associated morbidity is alleviated, repeated mass drug administration (MDA) at regular intervals depending on the population prevalence has been recommended by the WHO (Hotez et al. 2006; Kabatereine et al. 2007; WHO, 2013). Thus, it is important that sensitive diagnostic tools are applied to determine infection levels in the population.

Egg count in urine (parasitology) is the widely accepted approach for quantifying $S$. haematobium infection levels in a population (WHO, 1998; Pasvol and Hoffman, 2001; Kinkel et al. 2012). However, the parasitological method is less sensitive in light infections (Doenhoff et al. 2004; Bergquist et al. 2009). Furthermore, parasitology does not diagnose pre-patent or single-sex infections where there is no egg production (Mutapi, 2011). Several additional methods aimed at improving the diagnosis of schistosomiasis have been evaluated (Stothard et al. 2013), although the focus has been mainly on Schistosoma mansoni (Sorgho et al. 2005; de Noya et al. 2007; Stothard et al. 2011a). Examples of additional diagnostic methods include antibody detection (Sorgho et al. 2005; de Noya et al. 2007; Smith et al. 2012), dipstick detection of haematuria (Adesola et al. 2012; King and Bertsch, 2013) and use of reported questionnaires about presence of haematuria (Lengeler et al. 2002; Clements et al. 2008). There is currently a paucity of studies comparing different methods of detecting infection in preschool-aged children. The elegant dipstick metaanalysis study recently published by King and Bertsch (2013) highlights the need for more investigations on different methods for detecting infection in preschool-aged children.

The first aim of our study was to compare levels of $S$. haematobium infection determined by the parasitological method with infection detected via the serological technique and their implications for the WHO recommended treatment regimens for this study population. Dipstick microhaematuria was also used as an additional tool to the parasitological method on a subset of this study population to detect $S$. haematobium infection. The second aim of this study was to determine infection levels in preschoolaged children in comparison to primary school-aged children to elucidate the implications of these levels of infection for childhood health and their inclusion in the current control programmes.

MATERIALS AND METHODS

\section{Ethical approval and consent}

The study received ethical and institutional approval from the University of Zimbabwe and the Research Council of Zimbabwe. Permission to conduct the work in this province was obtained from the Provincial Medical Director, the District Educational Officer and Heads of schools in the study area. Project aims and procedures were fully explained to the community, primary school-aged children, teachers and parents/guardians in the local language, Shona. Written informed consent/assent was obtained from parents/guardians prior to enrolment of children into the study. The children were recruited into the study on a voluntary basis and were free to withdraw at any time with no further obligation. Children in this study were offered treatment with the standard dose of praziquantel administered by the local physician.

\section{Study area and population}

The study was conducted in two rural villages in Murewa district, in the north-east of Zimbabwe $\left(31^{\circ} 90^{\prime} \mathrm{E} ; 17^{\circ} 63^{\prime} \mathrm{S}\right)$. The area is a high $S$. haematobium transmission area according to the WHO classification of having a prevalence of infection $>50 \%$ (WHO, 2002). Prevalence of S. mansoni and soil transmitted helminths (STH) is low in this area (Ndhlovu et al. 1996; Nausch et al. 2012). The children were recruited from crèches, early child development centres, preschools (typically for 3-5 years old) and local primary schools (for 6-10 years old). Parents/ guardians with children not attending any of the education programmes (e.g. children $<3$ years old) in the area were invited to report to the school centre for enrolment into the project.

\section{Study design}

The inclusion/exclusion criteria for this study were as follows: children should have (1) been lifelong residents of the study area; (2) had no prior history of anthelmintic treatment (the above two criteria were 
assessed by means of questionnaires administered to parents/guardians for all children); (3) had provided at least 3 urine samples for S. haematobium detection and 2 stool samples for STH and S. mansoni parasitological examination; (4) been negative for $S$. mansoni infection (21 children were excluded from the study based on this criterion); and (5) been negative for STH infections (no children were excluded based on this criteria as no STH were detected in any of the participants). A total of 438 children $(54.6 \%$ females and $48.9 \%$ males) with complete parasitological and serological data were available for investigation in this study (Table A1). Of the surveyed children, $224(51 \cdot 1 \%)$ resided in village 1 and $214(48 \cdot 9 \%)$ were residents of village 2 .

\section{Parasitology}

Urine samples collected on 3 consecutive days were examined microscopically for $S$. haematobium infection using the standard filtration method (Mott et al. 1982). Schistosoma mansoni infection was diagnosed from stool samples collected on 2 consecutive days using the Kato-Katz method (Katz et al. 1972). Children were designated infected with S. haematobium if at least one egg was detected in any of their urine samples and similarly for $S$. mansoni with a single egg detected in stool. The S. haematobium infection intensity was calculated using the arithmetic mean egg count per $10 \mathrm{~mL}$ of the collected urine samples. For very young children where it was difficult to obtain samples on the spot, the samples were collected overnight by parents/guardians using urine collection bags (Hollister 7511 U-Bag Urine Specimen Collector, Hollister Inc., Chicago, IL, USA) and stool samples were collected using disposal dippers.

\section{Serology}

Serum was obtained from up to $5 \mathrm{~mL}$ of venous blood collected from each child, frozen at $-20^{\circ} \mathrm{C}$ in the field and transferred to a $-80^{\circ} \mathrm{C}$ freezer in the laboratory, prior to shipment to the University of Edinburgh, UK and kept under storage at $-80^{\circ} \mathrm{C}$. Samples were thawed for the first time for use in this study. The sera were tested for IgM (Dako, UK) antibody responses directed against schistosome egg antigens using enzyme linked immunosorbent assays (ELISA). The ELISA were conducted in duplicate per plate as previously described (Mutapi et al. 1997; Imai et al. 2011). The results were expressed as the mean optical density (OD) value of the duplicate assay. IgM antibodies are produced early in an infection (Warrington et al. 2011) and previous studies have reported a positive association between anti-egg IgM antibody responses and schistosomiasis infection levels (Mutapi et al. 2003; Stothard et al. 2011a; Dawson et al. 2013). Thus, for this study we used anti-schistosome egg $\operatorname{IgM}$ antibody response as an additional diagnostic indicator for $S$. haematobium infection.

A total of 17 serum samples comprised of four serum samples from age-matched schistosome naïve European and 13 healthy Zimbabwean donors (schistosome infection-free and with no anomalies reported after clinical examination by the paediatrician) were used as controls to determine cut-off ELISA values for 'infection' status. The European samples were drawn from the Edinburgh anonymized clinical sample archive. The cut-offs were calculated using the formula: mean (OD) $+2 *$ standard deviations of the mean (S.D.). Children were classified as infected if their levels of parasite-specific antibody levels were greater than the cut-off value, and infection negative if equal or below the cut-off value.

\section{Dipstick microhaematuria}

Out of the 438 children, 190 (51 preschool-aged and 139 primary school-aged) children in this study population had their urine samples examined for microhaematuria detectable by Uristix ${ }^{\circledR}$ reagent strips (Plasmatec, UK) as an indicator for $S$. haematobium infection in addition to the parasitological and serological diagnostic methods (Table A2). No marked variability was noted in dipstick tests of the urine samples for each child collected on consecutive days, thus, only the dipstick test results for urine samples collected on the first day of the survey were used in this study. The levels of dipstick microhaematuria were first graded semiquantitatively as: negative $(-)$, single positive $\left(+; \approx 10\right.$ erythrocytes $\left.\mu \mathrm{L}^{-1}\right)$, double positive $(++$; $\approx 50$ erythrocytes $\left.\mu \mathrm{L}^{-1}\right)$ and strong positive $(+++$; $\approx 250$ erythrocytes $\mu \mathrm{L}^{-1}$ ) following the manufacturer's guidelines. In this study, a positive test for microhaematuria was indicative of the presence of $S$. haematobium infection, meaning that children with scores of a single + and above were scored as positive for microhaematuria. A random sample of 123 urine samples were tested using the Multistix ${ }^{\circledR}$ 10SG (Bayer, UK) in addition to the Uristix ${ }^{\circledR}$ test to assess for differences in the quality of dipsticks by manufacturer. A strong agreement between the dipstick results from the two manufacturers using the McNemar's test $(P<0 \cdot 001)$ was observed, hence no evidence of the influence of the dipstick source on test results was noted for this study population.

\section{Statistical analyses}

Statistical analyses were performed using $\mathrm{SAS}^{\circledR} 9.3$ (SAS Institute Inc., Cary, NC, USA) and R 3.0.1 (R Development Core Team, Vienna, Austria). Infection intensity was $\log$-transformed $\left(\log _{10}[\mathrm{egg}\right.$ 
Table 1. Summary results for infection intensity using egg count per $10 \mathrm{~mL}$ urine, IgM antibody response in optical densities (OD) directed against schistosome egg antigens with standard deviation of the mean (S.D.) and $t$-test (on transformed data for infection intensity) for mean difference between the two age groups

\begin{tabular}{|c|c|c|c|c|c|c|c|c|}
\hline Variable & Age group & $n$ & Mean (s.D.) & Median & Minimum & Maximum & $t$ & $P$ value \\
\hline Egg count & $\begin{array}{l}1-5 \text { years } \\
6-10 \text { years }\end{array}$ & $\begin{array}{r}97 \\
341\end{array}$ & $\begin{array}{r}9 \cdot 03(47 \cdot 53) \\
19 \cdot 78(76 \cdot 50)\end{array}$ & $\begin{array}{l}0 \cdot 00 \\
0 \cdot 00\end{array}$ & $\begin{array}{l}0 \cdot 00 \\
0 \cdot 00\end{array}$ & $\begin{array}{r}380 \cdot 33 \\
1013 \cdot 00\end{array}$ & $-4 \cdot 49$ & $<0.001$ \\
\hline Antibody level & $\begin{array}{l}1-5 \text { years } \\
6-10 \text { years }\end{array}$ & $\begin{array}{r}97 \\
341\end{array}$ & $\begin{array}{l}0.46(0 \cdot 31) \\
0.67(0 \cdot 33)\end{array}$ & $\begin{array}{l}0 \cdot 38 \\
0 \cdot 66\end{array}$ & $\begin{array}{l}0 \cdot 01 \\
0 \cdot 07\end{array}$ & $\begin{array}{l}1 \cdot 27 \\
2 \cdot 39\end{array}$ & $-5 \cdot 72$ & $<0.001$ \\
\hline
\end{tabular}

count +1$]$ ) to meet the underlying assumptions of parametric statistical tests. Pearson's partial correlation coefficient $(r)$ was used to measure the strength of the association between infection intensity and antibody levels, controlling for the effect of age. To investigate whether the mean antibody levels or mean infection intensity differed significantly between preschool and primary school-aged children, independent $t$ tests were used. The effect of sex, age group and village on the mean infection intensity and on the antibody levels was investigated using general linear regression models. To determine whether infection prevalence differed between the two age groups and that prevalence derived from parasitological data differed from that based on serological data, Chi-square $\left(\chi^{2}\right)$ tests were used.

\section{Age-dependent prevalence model}

Infection prevalence based on the binary response variables derived from parasitology and serology as a function of age, was estimated parametrically using the method of generalized linear regression modelling. Letting $n$ be the sample size under investigation, $a_{i}$ the age of the $i$ th child $(i=1, \ldots n)$ and $q(a)$ the proportion of infection-negative children at age $a$ in the study population. The prevalence, which is the probability of being infected at age $a$, is given by: $\pi(a)$ $=1-q(a)$ and estimated using the binary response variable $Y_{i}$ as follows: $\pi(a)=P\left(Y_{i}=1 \mid a_{i}\right)$. The generalized linear model with a complementary loglog link was applied to take into account the binary nature of the response (Mathei et al. 2006) and expressed parametrically as follows:

$$
\pi(a)=1-\exp \left(-\alpha a^{\beta}\right)
$$

where $\alpha$ is the intercept and $\beta$ is the slope, i.e. the coefficient representing the effect of age on the probability of being infection positive.

$P$ values less than $0 \cdot 05$ were considered statistically significant in this study.

\section{RESULTS}

\section{Infection intensity and antibody levels}

The observed overall mean $S$. haematobium infection intensity based on egg counts was $17 \cdot 40$ eggs/10 mL
Table 2. $F$ and $P$ values from general linear regression models to test for the difference in mean infection intensity (transformed using $\log 10[\mathrm{egg}$ count +1$]$ ) and IgM antibody response directed against schistosome egg antigens by sex and village, adjusting for the effect of age

\begin{tabular}{lll}
\hline \hline & Infection intensity & Antibody level \\
\hline Variable & $F(P$ value $)$ & $F(P$ value $)$ \\
Age (years) & $13 \cdot 92(<0 \cdot 001)$ & $47 \cdot 47(<0 \cdot 001)$ \\
Sex $(\mathrm{F}$ vs $\mathrm{M})$ & $2 \cdot 11(0 \cdot 147)$ & $1 \cdot 51(0 \cdot 220)$ \\
Village $(1$ vs 2$)$ & $0 \cdot 49(0 \cdot 483)$ & $0 \cdot 48(0 \cdot 491)$ \\
\hline \hline
\end{tabular}

urine (S.D. $=71 \cdot 20)$ and the overall mean antibody levels was $0.62 \mathrm{OD}$ (S.D. $=0 \cdot 34$ ). The mean infection intensity and antibody levels were significantly higher for the primary-school aged children compared with that for preschool-aged children as shown in Table 1. A large variability in egg counts was observed as indicated by the large S.D. of the mean for both age groups in Table 1. Based on parasitology, $7 \cdot 1 \%$ of all children participating in this study carried heavy infections ( $\geqslant 50$ eggs $/ 10 \mathrm{~mL}$ urine), $30 \cdot 4 \%$ had light infections (1-49 eggs/10 mL urine) and $62 \cdot 6 \%$ had no infection burden ( 0 eggs $/ 10 \mathrm{~mL}$ urine) according to the WHO classes of infection intensity (WHO, 2002). Among the preschool-aged children, $3 \cdot 1 \%$ had heavy infections, $15 \cdot 5 \%$ had light infections and $81 \cdot 4 \%$ had no infection and in primary schoolaged children, $8 \cdot 2 \%$ had heavy infections, $34 \cdot 6 \%$ had light infections and $57 \cdot 2 \%$ had no infection.

Both infection intensity $(r=0 \cdot 18 ; P<0.001)$ and antibody levels $(r=0.31 ; P<0.001)$ increased significantly with age. In addition, a positive correlation between infection intensity and antibody levels was found $(r=0.23 ; P<0 \cdot 001)$. Infection intensity or antibody levels were not associated with sex and village of origin allowing for the effect of age ('Table 2).

\section{Infection prevalence: parasitology vs serology}

The overall observed infection prevalence based on the two diagnostic techniques was as follows: parasitology, $37 \cdot 4 \% \quad(95 \%$ CI: $33 \cdot 0-42 \cdot 0 \%)$ and serology, $71 \cdot 5 \%(95 \% \mathrm{CI}: 67 \cdot 2-75 \cdot 7 \%)$ and these 


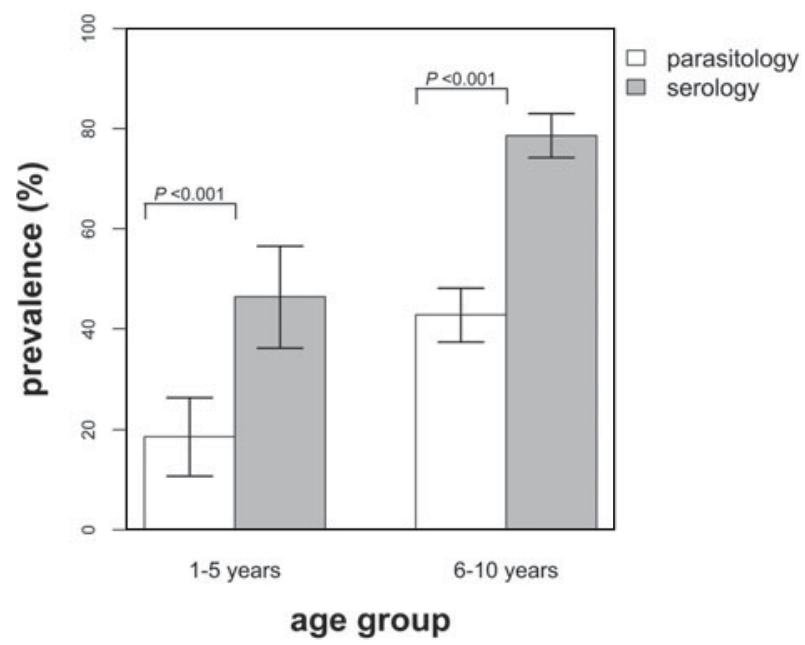

Fig. 1. Infection prevalence derived using parasitological and serological diagnostic methods by age group. The indicated bars are the $95 \%$ confidence intervals of the observed prevalence and the $P$ values test for the differences in prevalence between the diagnostic methods for each age group. White bars = prevalence based on parasitology and grey bars = prevalence based on serology.

differed significantly $\left(\chi^{2}=102 \cdot 12 ; \quad P<0 \cdot 001\right)$. In addition, the infection prevalence based on serology was found to be significantly higher than the prevalence derived from parasitology for both age groups (Fig. 1). No significant difference in infection prevalence between male and female children was observed (parasitology, $\chi^{2}=0.79 ; P=0.374$, and serology, $\left.\chi^{2}=0 \cdot 15 ; P=0 \cdot 703\right)$.

The proportion of children classified as infection negative using the parasitological technique in preschool-aged children was significantly lower $\left(\chi^{2}=4 \cdot 11 ; P=0 \cdot 043\right)$ compared with that in primary school-aged children (Fig. 2). For this study, only $16(3 \cdot 7 \%)$ children (10 female and 6 male, age $\geqslant 5$ years) were found egg positive but classified as infection negative using the serological diagnostic method. In addition, for 9 of these children, eggs were detected only in one urine sample, with a mean count of 4 eggs $/ 10 \mathrm{~mL}$ urine or less.

\section{Age-dependent prevalence profiles: parasitology vs serology}

The results for estimated regression coefficients and s.E. used to determine the age-dependent infection prevalence based on parasitological vs serological data were as follows: intercept, $\alpha: 0 \cdot 04$ (s.E. $=0.02$ ) vs $0 \cdot 12$ $($ S.E. $=0.05)$ and slope, $\beta: 1.23$ (s.E. $=0.27)$ vs 1.25 $($ s.E. $=0 \cdot 19)$. Infection prevalence increased with age in a similar pattern for both diagnostic methods, however the rate of increase for serology was higher compared with that of parasitology (Fig. 3). In addition, the infection prevalence derived using the

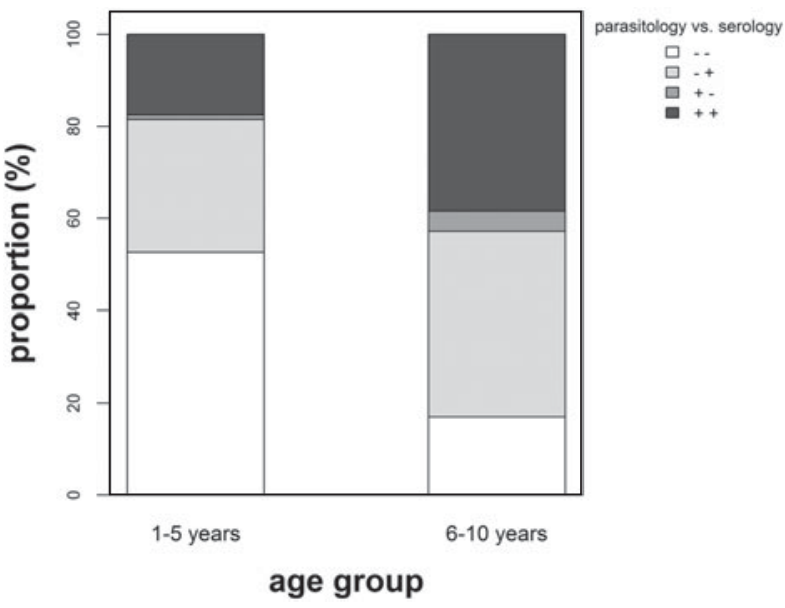

Fig. 2. Percentage proportion positive $(+)$ vs negative (-) children diagnosed using parasitological and serological methods by age group. White stack: $(--)=$ negative for both diagnostic methods ( $1-5$ years, $n=51 ; 6-10$ years, $n=58)$, light grey stack $(-+)=$ negative for parasitology but positive for serology ( $1-5$ years, $n=28$; $6-10$ years, $n=137)$, grey stack $(+-)=$ positive for parasitology but negative for serology ( $1-5$ years, $n=1$; $6-10$ years, $n=15$ ) and dark grey stack $(++)=$ positive for both diagnostic methods $(1-5$ years, $n=17$; $6-10$ years, $n=131)$.

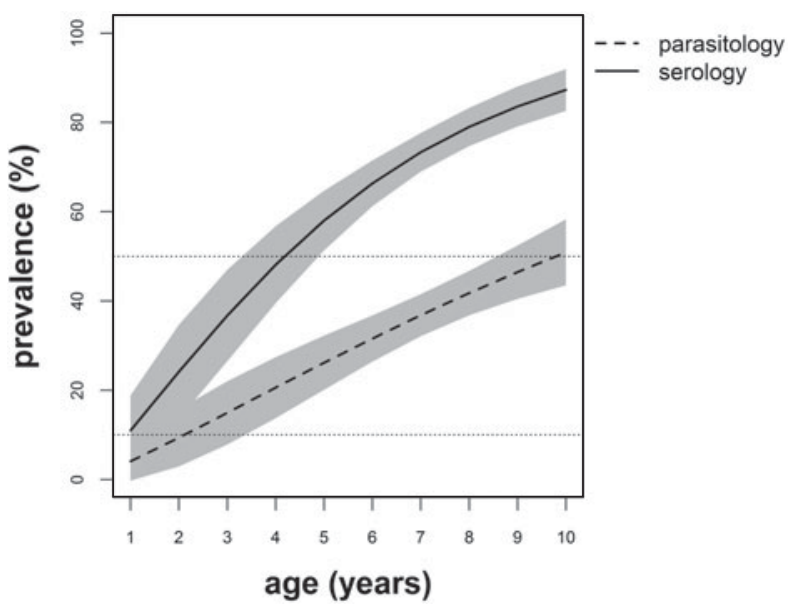

Fig. 3. Predicted age-related infection prevalence profiles derived from parasitological (dashed line) and serological (solid line) diagnostic methods. The grey shadings around the prevalence curves indicate the $95 \%$ confidence intervals. The horizontal dashed lines indicate the moderate $(10 \%)$ and high $(50 \%)$ infection-risk cut-offs for control regimens as defined by the World Health Organization (WHO, 2002).

serological technique was higher compared with the prevalence based on parasitological diagnostic method and this discrepancy increased with age (Fig. 3). The infection levels for primary schoolaged children based on serology belonged to the highrisk WHO category (prevalence $\geqslant 50 \%$ ) compared with the moderate-risk category implied by the parasitological diagnostic method. 


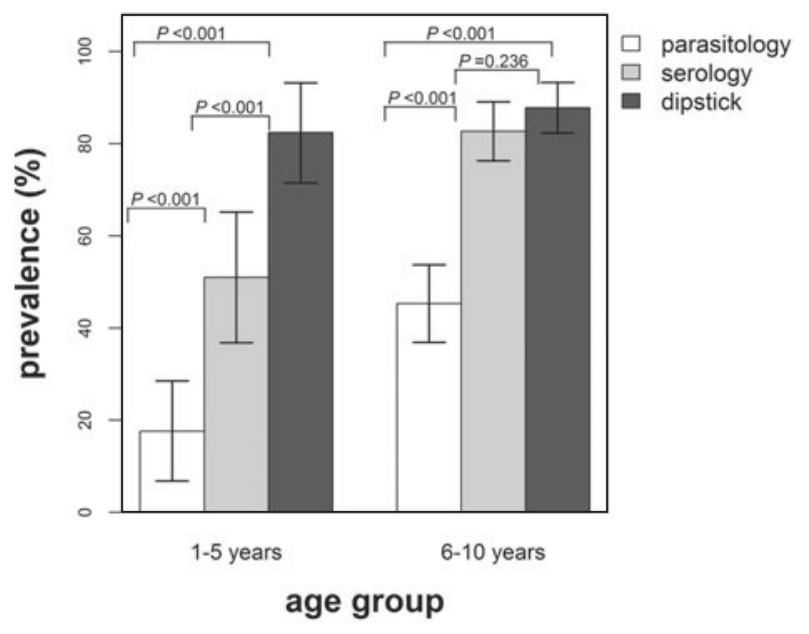

Fig. 4. Infection prevalence derived using parasitological, serological and dipstick microhaematuria diagnostic methods by age group for a subset of the study population $(n=190)$. The indicated bars are the $95 \%$ confidence intervals of the observed prevalence and the $P$-values test for the differences in prevalence between the diagnostic methods for each age group. White bars $=$ prevalence based on parasitology, grey bars $=$ prevalence based on serology and dark grey bars $=$ prevalence based on dipstick microhaematuria .

\section{Dipstick microhaematuria diagnostic method}

Infection prevalence derived using the dipstick microhaematuria test was compared with the infection prevalence determined using the parasitological diagnostic method on 190 children (Fig. 4). The overall infection prevalence derived from the dipstick microhaematuria in this subset of the study population was $86 \cdot 3 \%(95 \% \mathrm{CI}: 81 \cdot 9-91 \cdot 7 \%)$ compared with $37 \cdot 9 \%(95 \% \mathrm{CI}: 30 \cdot 9-44 \cdot 9 \%)$ based on parasitology and $74 \cdot 2 \%$ (95\% CI: $67 \cdot 9-80 \cdot 5 \%)$ derived from the serological diagnostic method. Furthermore, infection prevalence based on dipstick microhaematuria was significantly higher compared with prevalence based on parasitology for both age groups. It was further noted that none of the egg-positive children were diagnosed as infection negative using dipstick microhaematuria and $4(2.9 \%)$ primary school-aged children were found egg positive but with no microhaematuria detected in urine.

\section{DISCUSSION}

Following successful advocacy by the World Health Assembly (WHA, 2001), repeated MDA has become the key control strategy to combat schistosomiasis (WHO, 2002, 2013), with frequency of treatment dependent on the pre-determined infection prevalence (WHO, 2002). However, taking into consideration the reduced sensitivity of the parasitological diagnostic technique in children carrying light infections (Engels et al. 1997; Coulibaly et al. 2013), it is imperative that additional sensitive diagnostic tools are incorporated to improve the determination of infection levels. In this study we compared levels of $S$. haematobium infection obtained by the parasitological (egg count) method to the serological technique. In addition, these infection levels were compared between preschool and primary schoolaged children to elucidate the need for inclusion of the neglected preschool age group into control programmes. The implications of infection levels determined in this study for the WHO recommended MDA regimens were also investigated.

In agreement with other studies using different diagnostic tools (Kahama et al. 1998; Kanamura et al. 2002; Lengeler et al. 2002; van Dam et al. 2004), infection levels (infection intensity and prevalence) increased significantly with age in this study. Unsurprisingly, infection intensity was positively correlated with anti-egg IgM antibody levels, since children accumulate infection with the associated increase in exposure to schistosome antigens (Stothard et al. 2011b). More importantly, the results of this study revealed significant infection prevalence in preschool-aged children, further concurring with findings from recent studies on the infection burden in this age group (Garba et al. 2010; SousaFigueiredo et al. 2010; Mutapi et al. 2011; Stothard et al. 2011a). These findings implicate a risk of preschool-aged children developing severe pathology due to chronic infection if left untreated (Stothard et al. 2011b; Ekpo et al. 2012). Hence the inclusion of these children in control programmes should be considered fundamental for improved and balanced childhood health (Garba et al. 2010).

This study revealed, in contrast with serology, that the parasitological technique approach underestimated infection prevalence in both age groups. These findings are indicative of reduced sensitivity of the parasitological technique since the majority of children in our study population carried light infection. In addition, following the WHO guidelines (WHO, 2002), infection prevalence derived from the serological method suggested a more frequent treatment intervention for this study population compared with that implicated by the parasitological technique. These findings further demonstrate that the use of different diagnostic techniques can be of importance in decision-making about suitable control strategies to implement. The WHO system is based upon parasitology, and was developed before the contribution of light infections (not detected via egg counts) to pathology was fully realized. The combination of additional diagnostics which can detect low infection levels and better definition of morbidity arising from low infections in S. haematobium infections (as recently summarized by King and Bertsch, 2013) support the current efforts for including preschool-aged children in schistosomiasis control programmes (Stothard et al. 2013). 
The small proportion of schistosome egg-positive children in this study who were classified as infection negative using the serological technique can theoretically be attributable to two reasons: (1) contamination of the urine samples (Mutapi, 2011), which can occur as a result of instruments not being thoroughly cleaned or urine contamination with stool, especially for young female children; and (2) individual variability in mounting an immune response against the parasite antigens (Stothard et al. 2011a).

Similar patterns of age-dependent infection prevalence profiles were observed for both diagnostic methods, indicative of early exposure to infection and the accumulation of infection as children grow older (Garba et al.2010; Stothard et al.2011a). Overall, the estimated age-dependent prevalence based on serology was higher compared with that derived from parasitology, and this discrepancy between infection levels obtained from the two diagnostic methods also increased with age. Consequentially, the observed age-prevalence patterns indicated that the required intervention strategies varied with age.

The use of dipstick microhaematuria in this study detected higher prevalence of infection equally for both preschool and primary school-aged children in comparison to the parasitological method. These findings highlighted the usefulness of dipstick microhaematuria as an additional diagnostic tool in children carrying light infections, in agreement with findings from other recent studies (King and Bertsch, 2013). Haematuria due to glomerular causes has been reported in children (Meyers, 2004), thus caution should be exercised when interpreting the high prevalence of microhaematuria in preschool-aged children. Further studies are needed to elucidate levels of haematuria attributable to schistosome infection in this age group. French et al. (2007) recommended comparison of dipsticks sourced from different manufacturers to assess the effect of quality on the test results. In this study we used dipsticks sourced from two different companies (Uristix ${ }^{\circledR}$ from Plasmatec and Multistix ${ }^{\circledR}$ from Bayer) and they gave comparable results, supporting the robustness of our findings.

\section{CONCLUSION}

In conclusion, this study showed significant S. haematobium infection levels among untreated preschool and primary school-aged children who were life-long residents of an endemic area. Infection intensity and prevalence increased rapidly from early childhood, highlighting the need for treatment of the preschool-aged children. This study further highlighted the essential need for incorporating preschool-aged children into control programmes for the health benefits of treatment currently being offered to their older counterparts and thus prevent creating a childhood health inequity (Mutapi et al.
2011; Stothard et al. 2011b, 2013). Infection prevalence based on serology suggested a more frequent MDA regimen to that implied by the parasitological technique. We reiterate the importance of using sensitive diagnostic methods to improve accuracy in estimating true infection prevalence as this has implications on the required MDA regimen for the population. In our study, serology was highlighted as a valuable sensitive diagnostic tool that could be applied in conjunction with the parasitological technique. The findings of this study revealed that dipstick microhaematuria was equally sensitive in diagnosing infection in both preschool and primaryschool aged children. Further evaluation of detection of microhaematuria using dipsticks as an additional diagnostic tool for $S$. haematobium infection in preschool-aged children is recommended.

\section{ACKNOWLEDGEMENTS}

We are grateful for the cooperation of the Ministry of Health and Child Welfare in Zimbabwe, the Provincial Medical Director of Mashonaland East, residents, teachers, parents and children from Chingwaru and Chingono. We extend a special thank you to the participants of this study. We also thank members of the National Institute for Health Research (Zimbabwe) and the University of Zimbabwe for technical support. This work was first presented as a poster at the British Society for Parasitology Autumn Symposium 2013 on 'Advances in Diagnostics for Infectious Diseases'.

\section{FINANCIAL SUPPORT}

The study received financial support from the World Health Organization, the Wellcome Trust, UK (Grant number WT082028MA) and the Thrasher Research Fund (Grant number 02832-5) and the Schistosome Control Initiative. The Edinburgh Clinical Sample Archive is supported by a grant from the Wellcome Trust to the Centre for Immunity, Infection and Evolution.

\section{REFERENCES}

Adesola, H., Uduak, N., Olajumoke, M., Roseangela, N., Chiaka, A., Sunday, A., Oyetunde, S., Ayodele, J. and Alex, O. (2012). Urine turbidity and microhaematuria as rapid assessment indicators for Schistosoma haematobium infection among school children in endemic areas. American Fournal of Infectious Diseases 8, 60-64.

Bergquist, R., Johansen, M. V. and Utzinger, J. (2009). Diagnostic dilemmas in helminthology: what tools to use and when? Trends in Parasitology 25, 151-156.

Clements, A. C. a., Barnett, A. G., Nyandindi, U., Lwambo, N. J. S., Kihamia, C. M. and Blair, L. (2008). Age and gender effects in selfreported urinary schistosomiasis in Tanzania. Tropical Medicine and International Health 13, 713-721.

Coulibaly, J. T., N'Gbesso, Y. K., Knopp, S., Keiser, J., N'Goran, E. K. and Utzinger, J. (2012). Efficacy and safety of praziquantel in preschoolaged children in an area co-endemic for Schistosoma mansoni and S. haematobium. PLoS Neglected Tropical Diseases 6, e1917.

Coulibaly, J. T., N'Goran, E. K., Utzinger, J., Doenhoff, M. J. and Dawson, E. M. (2013). A new rapid diagnostic test for detection of antiSchistosoma mansoni and anti-Schistosoma haematobium antibodies. Parasites and Vectors 6, 29.

Dawson, E. M., Sousa-Figueiredo, J. C., Kabatereine, N. B., Doenhoff, M. J. and Stothard, J. R. (2013). Intestinal schistosomiasis in preschool-aged children of Lake Albert, Uganda: diagnostic accuracy of a rapid test for detection of anti-schistosome antibodies. Transactions of the Royal Society of Tropical Medicine and Hygiene 107, 639-647. 
de Noya, A. B., Ruiz, R., Losada, S., Colmenares, C., Contreras, R., Cesari, I. M. and Noya, O. (2007). Detection of schistosomiasis cases in low-transmission areas based on coprologic and serologic criteria: the Venezuelan experience. Acta Tropica 103, 41-49.

Doenhoff, M. J., Chiodini, P. L. and Hamilton, J. V. (2004). Specific and sensitive diagnosis of schistosome infection: can it be done without antibodies? Trends in Parasitology 20, 1471-1492.

Doenhoff, M. J., Cioli, D. and Utzinger, J. (2008). Praziquantel: mechanisms of action, resistance and new derivatives for schistosomiasis. Current Opinion in Infectious Diseases 21, 659-667.

Ekpo, U. F., Oluwole, A.S., Abe, E. M., Etta, H. E., Olamiju, F. and Mafiana, C. F. (2012). Schistosomiasis in infants and pre-school-aged children in sub-Saharan Africa: implication for control. Parasitology 10, $1-7$.

Engels, D., Sinzinkayo, E., de Vlas, S. J. and Gryseels, B. (1997). Intraspecimen fecal egg count variation in Schistosoma mansoni infection. American Fournal of Tropical Medicine and Hygiene 57, 571-577.

French, M. D., Rollinson, D., Basanez, M. G. and Gryseels, B. (2007). School-based control of urinary schistosomiasis on Zanzibar, Tanzania: monitoring micro-haematuria with reagent strips as a rapid urological assessment. Fournal of Pediatric Urology 3, 364-368.

Garba, A., Barkiré, N., Djibo, A., Lamine, M.S., Sofo, B., Gouvras, A. N., Bosqué-Oliva, E., Webster, J. P., Stothard, J. R., Utzinger, J. and Fenwick, A. (2010). Schistosomiasis in infants and preschool-aged children: infection in a single Schistosoma haematobium and a mixed $S$. haematobium-S. mansoni foci of Niger. Acta Tropica 115, 212-219

Gryseels, B., Polman, K., Clerinx, J. and Kestens, L. (2006). Human schistosomiasis. Lancet 368, 1106-1118.

Hotez, P.J., Bundy, D. A. P., Beegle, K., Brooker, S., Drake, L., de Silva, N., Montresor, A., Engels, D., Jukes, M., Chitsulo, L., Chow, J., Laxminarayan, R., Michaud, C., Bethony, J., CorreaOliveira, R., Shuhua, X., Fenwick, A. and Savioli, L. (2006). Infections: soil-transmitted helminth infections and schistosomiasis. In Disease Control Priorities in Developing Countries (ed. Jamison, D. T., Breman, J. G., Measham, A. R., Alleyne, G., Claeson, M., Evans, D. B., Jha, P., Mills, A. and Musgrove, P.), pp. 467-482. Oxford University Press, New York, NY, USA.

Imai, N., Rujeni, N., Nausch, N., Bourke, C. D., Appleby, L. J., Cowan, G., Gwisai, R., Midzi, N., Cavanagh, D., Mduluza, T., Taylor, D. and Mutapi, F. (2011). Exposure, infection, systematic cytokine levels and antibody responses in young children concurrently exposed to schistosomiasis and malaria. Parasitology 138, 1519-1533.

Kabatereine, N. B., Brooker, S., Koukounari, A., Kazibwe, F., Tukahebwa, E. M., Fleming, F. M., Zhang, Y., Webster, J.P., Stothard, J. R. and Fenwick, A. (2007). Impact of a national helminth control programme on infection and morbidity in Ugandan schoolchildren. Bulletin of the World Health Organization 85, 91-99.

Kahama, I., Nibbeling, H., van Zeyl, R. J., Vennervald, B. J., Ouma, J. H. and Deelder, M. (1998). Detection and quantification of soluble egg antigen in urine of Schistosoma haematobium-infected children from Kenya. American Fournal of Tropical Medicine and Hygiene 59, 769-774

Kanamura, H. Y., Silva, R. M., Chiodelli, S. G., Glasser, C. M. and Dias, L. C. (2002). IgM-immunofluorescence test as a diagnostic tool for epidemiologic studies of schistosomiasis in low endemic areas. Memórias do Instituto Oswaldo Cruz 97, 485-489.

Katz, N., Chaves, A. and Pellegrino, J. (1972). A simple device for quantitative stool thick-smear technique in Schistosomiasis mansoni. Revista do Instituto de Medicina Tropical de São Paulo 14, 397-400.

King, C. H. and Bertsch, D. (2013). Meta-analysis of urine heme dipstick diagnosis of Schistosoma haematobium infection, including low-prevalence and previously treated populations. PLoS Neglected Tropical Diseases 7, e2431.

Kinkel, H.F., Dittrich, S., Baumer, B. and Weitzel, T. (2012). Evaluation of eight serological tests for diagnosis of imported schistosomiasis. Clinical Vaccine Immunology 19, 948-953.

Lengeler, C., Utzinger, J. and Tanner, M. (2002). Questionnaires for rapid screening of schistosomiasis in sub-Saharan Africa. Bulletin of the World Health Organization 80, 235-242.

Mathei, C., Shkedy, Z., Denis, B., Kabali, C., Aerts, M., Molenberghs, G., Van Damme, P. and Buntinx, F. (2006). Evidence for a substantial role of sharing of injecting paraphernalia other than syringes/needles to the spread of hepatitis $\mathrm{C}$ among injecting drug users. Fournal of Viral Hepatitis 13, 560-570.

Meyers, K. E. (2004). Evaluation of hematuria in children. Urologic Clinics of North America 31, 559-573, x.
Mott, K., Baltes, R., Bambagha, J. and Baldassini, B. (1982). Field studies of a reusable polyamide filter for detection of Schistosoma haematobium eggs by urine filtration. Tropenmedizin und Parasitologie 33 227-228.

Muller, I., Coulibaly, J. T., Furst, T., Knopp, S., Hattendorf, J. Krauth, S. J., Stete, K., Righetti, A. A., Glinz, D., Yao, A. K. Puhse, U., N'Goran, E. K. and Utzinger, J. (2011). Effect of schistosomiasis and soil-transmitted helminth infections on physical fitness of school children in Cote d'Ivoire. PLoS Neglected Tropical Diseases 5, e1239.

Mutapi, F. (2011). Improving diagnosis of urogenital schistosome infection. Expert Review of Anti-infective Therapy 9, 863-865.

Mutapi, F., Ndhlovu, P. D., Hagan, P. and Woolhouse, M. E. (1997). A comparison of humoral responses to Schistosoma haematobium in areas with low and high levels of infection. Parasite Immunology 19, 255-263.

Mutapi, F., Hagan, P., Woolhouse, M. E. J., Mduluza, T. and Ndhlovu, P. D. (2003). Chemotherapy-induced, age-related changes in antischistosome antibody responses. Parasite Immunology 25, 87-97.

Mutapi, F., Rujeni, N., Bourke, C., Mitchell, K., Appleby, L., Nausch, N., Midzi, N. and Mduluza, T. (2011). Schistosoma haematobium treatment in 1-5 year old children: safety and efficacy of the antihelminthic drug praziquantel. PLoS Neglected Tropical Diseases 5 , e1143

Nausch, N., Bourke, C. D., Appleby, L. J., Rujeni, N., Lantz, O., Trottein, F., Midzi, N., Mduluza, T. and Mutapi, F. (2012). Proportions of CD4 + memory T cells are altered in individuals chronically infected with Schistosoma haematobium. Scientific Reports 2, 472.

Ndhlovu, P., Cadman, H., Vennervald, B. J., Christensen, N. O., Chidimu, M. and Chandiwana, S. K. (1996). Age-related antibody profiles in Schistosoma haematobium infections in a rural community in Zimbabwe. Parasite Immunology 18, 181-191.

Pasvol, G. and Hoffman, S. L. (2001). Schistosomiasis. Imperial College Press, London, UK.

Sady, H., Al-Mekhlafi, H. M., Mahdy, M. A., Lim, Y. A., Mahmud, R. and Surin, J. (2013). Prevalence and associated factors of schistosomiasis among children in Yemen: implications for an effective control programme. PLoS Neglected Tropical Diseases 7, e2377.

Smith, H., Doenhoff, M., Aitken, C., Bailey, W., Ji, M., Dawson, E., Gilis, H., Spence, G., Alexander, C. and van Gool, T. (2012) Comparison of Schistosoma mansoni soluble cercarial antigens and soluble egg antigens for serodiagnosing schistosome infections. PLoS Neglected Tropical Diseases 6, e1815.

Sorgho, H., Bahgat, M., Poda, J. N., Song, W., Kirsten, C., Doenhoff, M. J., Zongo, I., Ouedraogo, J. B. and Ruppel, A. (2005). Serodiagnosis of Schistosoma mansoni infections in an endemic area of Burkina Faso: performance of several immunological tests with different parasite antigens. Acta Tropica 93, 169-180.

Sousa-Figueiredo, J. C., Basanez, M. G., Mgeni, A. F., Khamis, I. S., Rollinson, D. and Stothard, J. R. (2008). A parasitological survey, in rura Zanzibar, of pre-school children and their mothers for urinary schistosomiasis, soil-transmitted helminthiases and malaria, with observations on the prevalence of anaemia. Annals of Tropical Medicine and Parasitology 102, 679-692.

Sousa-Figueiredo, J. C., Pleasant, J., Day, M., Betson, M., Rollinson, D., Montresor, A., Kazibwe, F., Kabatereine, N. B. and Stothard, J. R. (2010). Treatment of intestinal schistosomiasis in Ugandan preschool children: best diagnosis, treatment efficacy and sideeffects, and an extended praziquantel dosing pole. International Health 2 103-113.

Stothard, J. R. and Gabrielli, A.-F. (2007). Schistosomiasis in African infants and preschool children: to treat or not to treat? Trends in Parasitology, 23, 83-86.

Stothard, J. R., Sousa-Figueiredo, J. C., Betson, M., Adriko, M., Arinaitwe, M., Rowell, C., Besiyge, F. and Kabatereine, N. B. (2011a). Schistosoma mansoni infections in young children: when are schistosome antigens in urine, eggs in stool and antibodies to eggs first detectable? PLoS Neglected Tropical Diseases 5, e938.

Stothard, J.R., Sousa-Figueiredo, J.C., Betson, M., Green, H. K., Seto, E.Y.W., Garba, A., Sacko, M., Mutapi, F., Vaz Nery, S. Amin, M. A., Mutumba-Nakalembe, M., Navaratnam, A., Fenwick, A., Kabatereine, N. B., Gabrielli, A. F. and Montresor, A. (2011b). Closing the praziquantel treatment gap: new steps in epidemiological monitoring and control of schistosomiasis in African infants and preschool-aged children. Parasitology 138, 1593-1606.

Stothard, J. R., Sousa-Figueiredo, J. C., Betson, M., Bustinduy, A. and Reinhard-Rupp, J. (2013). Schistosomiasis in African infants and preschool children: let them now be treated! Trends in Parasitology 29,197-205. van Dam, G. J., Wichers, J.H., Ferreira, T. M., Ghati, D., van Amerongen, A. and Deelder, A. M. (2004). Diagnosis of 
schistosomiasis by reagent strip test for detection of circulating cathodic antigen. Fournal of Clinical Microbiology 42, 5458-5461.

van der Werf, M.J., de Vlas, S. J., Brooker, S., Looman, C. W., Nagelkerke, N. J., Habbema, J. D. and Engels, D. (2003). Quantification of clinical morbidity associated with schistosome infection in sub-Saharan Africa. Acta Tropica 86, 125-139.

Vennervald, B. J., Kahama, I. and Reimert, C. M. (2000). Assessment of morbidity in Schistosoma haematobium infection: current methods and future tools. Acta Tropica 77, 81-89.

Warrington, R., Watson, W., Kim, H. L. and Antonetti, F. R. (2011). An introduction to immunology and immunopathology. Allergy, Asthma and Clinical Immunology 7 (Suppl. 1), S1.

World Health Assembly (2001). Schistosomiasis and Soil-Transmitted Helminth Infections. WHA A54/VR/9. World Health Organization, Geneva, Switzerland.

World Health Organization (1998). Report of the WHO Informal Consultation on Schistosomiasis Control. WHO/CDS/CPC/SIP/99.2. World Health Organization, Geneva, Switzerland.
World Health Organization (2002). Prevention and Control of Schistosomiasis and Soil-Transmitted Helminthiasis. WHO Technical Report Series No. 912. World Health Organization, Geneva, Switzerland.

World Health Organization (2010). Working to Overcome the Global Impact of Neglected Tropical Diseases. WHO/HTM/NTD/2010.1. World Health Organization, Geneva, Switzerland.

World Health Organization (2011). Report of a Meeting to Review the Results of Studies on the Treatment of Schistosomiasis in Preschool-age Children. WHO/HTM/NTD/PCT/2011.7. World Health Organization, Geneva, Switzerland.

World Health Organization (2012). Research Priorities for Helminth Infections. WHO Technical Series No. 972. World Health Organization, Geneva, Switzerland.

World Health Organization (2013). Schistosomiasis: Progress Report 2001-2011 and Strategic Plan 2012-2020. WHO/HTM/NTD/PCT/ 2013.2. World Health Organization, Geneva, Switzerland.

\section{APPENDIX}

Table A1. Description of the different sample sizes of the study population (total, $n=438$ ) for parasitology and serology data by age group

\begin{tabular}{llccr}
\hline \hline & & \multicolumn{2}{c}{ Serology } & \\
\cline { 3 - 4 } Age group & Parasitology & Negative & Positive & Total \\
\hline 1-5 years & Negative & 51 & 28 & 79 \\
& Positive & 1 & 17 & 18 \\
6-10 years & Negative & 58 & 137 & 195 \\
& Positive & 15 & 131 & 146 \\
& Total & 125 & 313 & 438 \\
\hline \hline
\end{tabular}

Table A2. Description of the different sample sizes of the subset (total, $n=190$ ) of the study population for parasitology, serology and dipstick microhaematuria data by age group

\begin{tabular}{|c|c|c|c|c|c|}
\hline \multirow[b]{2}{*}{ Age group } & \multirow[b]{2}{*}{ Parasitology } & \multicolumn{2}{|l|}{ Serology } & \multicolumn{2}{|c|}{ Dipstick microhaematuria } \\
\hline & & Negative & Positive & Negative & Positive \\
\hline \multirow[t]{2}{*}{$1-5$ years } & Negative & 25 & 17 & 9 & 33 \\
\hline & Positive & 0 & 9 & 0 & 9 \\
\hline \multirow[t]{3}{*}{$6-10$ years } & Negative & 22 & 54 & 13 & 63 \\
\hline & Positive & 2 & 61 & 4 & 59 \\
\hline & Total & 49 & 141 & 26 & 164 \\
\hline
\end{tabular}

